\title{
Heterogeneity Aware Clustered WSN using Multi-hop Communication
}

\author{
Abhilasha Pandey \\ M.TechStudent, \\ Department of ECE \\ Sagar Institute of Science and \\ Technology Bhopal India
}

\author{
Nitin Muchhal, Md Abdulla \\ Associate Professor, \\ Department of ECE \\ Sagar Institute of Science and \\ Technology, Bhopal India
}

\author{
Ravi Shankar Mishra \\ Professor \& Head, \\ Department of ECE \\ Sagar Institute of Science and \\ Technology, Bhopal India
}

\begin{abstract}
Energy in wireless sensor network is a recent topic of research. To organize the network such a way that should minimize energy dissipation and maximize network stability period and network lifetime. In this paper, a distributed, randomized Heterogeneity Aware Clustering Algorithm (HACA) to organize the sensors in a wireless sensor network into clusters has been proposed.HACA is very simple to implement in the sensor nodes. This is a dynamic protocol, it will work automatically over no prior distribution of the levels of energy in the nodes is to conserve.HACA algorithm alsogeneratea hierarchy of cluster heads and observe that the energy savings increase with the number of levels in the hierarchy. Results in stochastic geometry are used to derive solutions for the values of the parameters of proposed algorithm that minimize the total energy spent in the network when all sensors report data through the cluster heads to the processing center.
\end{abstract}

\section{Keywords}

HACA Algorithm, Asystem design, simulation by using mat lab.

\section{INTRODUCTION}

Wireless Sensor Network (WSN) is the network of tiny sized sensor nodes. Due to the limited size, sensor node is limited in processing, memory and energy. The limitation of energy and memory in the sensor node create challenges for routing in WSN. Recently clustering increases interest for energy efficiency in WSNs. A sensor node represents all other sensor nodes in the cluster called cluster head and collects sensed data from them. The load on cluster head is larger than normal sensor node there sincethree types of sensor node, first is super heterogeneous (more energy than normal and advance sensor node) sensor node, second is advance heterogeneous (more energy than normal sensor node) sensor node and third is normal sensor node is used in this paper. To balance the energy consumption among sensor nodes in the network, the cluster head should be rotated among all sensor nodes, but super sensor node should be selected cluster head more time than advance sensor node and normal sensor node and same for an advance sensor node. This will increases the stability period of the sensor network. In this paper, HACA elects cluster head based on residual power (energy) of the sensor nodes. In WSNs data reporting to the base station over long distance consumed large amount of energy that reduce the lifetime of the network. For that a new multihop approach for inter cluster communication is proposed. The simulation results show that HACA conserves more energy in data reporting compared to the well-known exiting clustering algorithms SEP and EEHC.

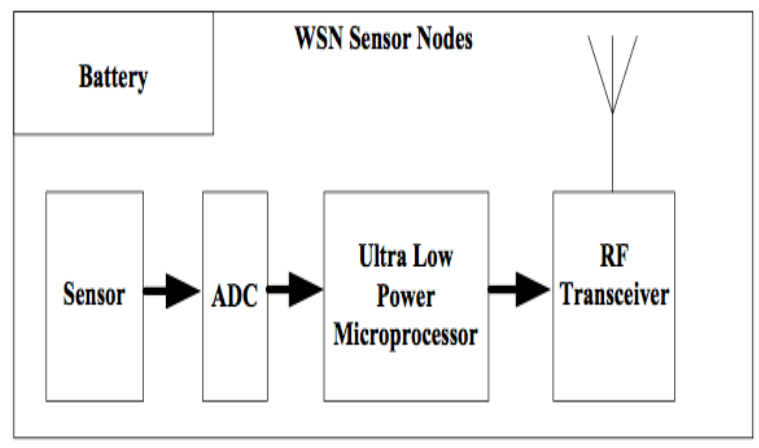

Fig 1: Block Diagram of Sensor Node

HACA also prolongs the network stability period and lifetime than SEP and EEHC protocols.

\section{RELATED WORK}

Heinzelman, Wendi Beth. et al. [1] have proposed Low Energy Adaptive Clustering Hierarchy LEACH includes distributed cluster formation, local processing to reduce global communication, and randomized rotation of the cluster-heads.

Younis, Ossama, and Sonia Fahmy.et al. [2] has proposed Hybrid Energy-Efficient Distributed Clustering HEED uses an iterative cluster formation algorithm, where sensors assign themselves a "cluster head probability" that is a function of their residual energy and a "communication cost" that is a function of neighbor proximity. This procedure iterates, with each sensor, increasing its cluster head probability in each iteration until the cluster head probability is one and the sensor declares itself a "final cluster head" for this cycle. The advantages of HEED are that nodes only require local (neighborhood) information to form the clusters, the algorithm terminates in $\mathrm{O}$ (1) iterations, the algorithm guarantees that every sensor is part of just one cluster, and the cluster heads are well-distributed.

Georgios S. et. Al [3] proposed a heterogeneity aware protocol is called SEP, in that election probability is weighted by the initial energy of a node relative to that of other nodes in the mesh.This extends the time interval before the expiry of the first node, which is important for many applications where the feedback from the sensor network must be reliable Training starts with a single cluster with its heart as the mean of the data [4]. This cluster is split into two and the means of the new clusters are iteratively trained. These two clusters are again split and the process continues until the designated number of clusters is obtained.

Wang, Guitang, et al. [6] have presented it selects cluster head with taking into account the energy and multi-hop combined 
with simple hop in the cluster. The Clustering Algorithm for Wireless Sensor Networks Based on Multi-Hop between Clusters. It selects cluster head with taking into account the energy and multi-hop mixed with simple hop in the bunch.Simulation results indicate that compared to LEACH, $\mathrm{MH}-\mathrm{LEACH}$ has better effect on extending the network lifetime and improving the energy usage rate.

Gupta, Gaurav, and Mohamed Younis. et al. [7] In this paper they suggest an algorithm to network these sensors into well define clusters with less-energy-constrained gateway nodes acting as cluster heads, and balance load among these gateways.

Kumar, Dilip, Trilok C. Aseri, and R. B. Patel et al. [8] Inthis papershow the impact of heterogeneity of nodes in terms of their energy in wireless sensor networks that are hierarchically clustered. The authors introduce an energy efficient heterogeneous clustered scheme for wireless sensor networks based on weighted election probabilities of each client to get a cluster head according to the residual energy in each client.As sensor network totally relies on battery power, the primary objective of maximizing the lifetime of the network is to conserve battery power or vitality. In sensor network, the energy is mainly consumed for three purposes: data transmission, signal processing, and hardware operation

The problem of many clustering approaches is that, when the sensor node is not taking in enough energy to transmit the information to the base station and becomes the cluster head.In these approaches, inter cluster communication is also being a large component of energy use of goods and services.Thus, there is an access to minimize the inter cluster communication without affecting the data reliability.This paper proposed a Heterogeneity Aware Clustering Algorithm which provides an energy effective network, which retain long time. In this protocol a new threshold has been proposedfor cluster head selection which select the cluster head based on sensor remaining energy. A new multihoped data reporting process; in which cluster head (normal node) send its data to nearest advance node $\mathrm{CH}$ and super node $\mathrm{CH}$ has been proposed. The advance node $\mathrm{CH}$ sends data to nearest super node $\mathrm{CH}$. It will save lots of energy in data transmission and increase stability period of the sensor network.

\section{PROPOSED APPROACH}

Energy efficient communication protocols proposed for wireless sensor networks. A method for managing cluster heads in such a way so that the network operational lifetime maximized. The HACA clustering process consists two sub part setup phase and steady state phase. In setup phase cluster head is selected on the basis of probabilistic threshold (based on residual energy). At the end of setup phase all the cluster heads are selected and formation of the cluster is accomplished. The steady state phase includes data aggregation and data reporting process. After the completion of sensing process, each $\mathrm{CH}$ receives data from its cluster member. It aggregated receive data and send it to the nearest super $\mathrm{CH}$ using multi-hop communication.

\subsection{Setup Phase}

The setup phase includes cluster head selection and cluster formation process.

\subsubsection{Cluster head selection}

In the cluster head selection process each sensor node chooses a random number between 0 and 1 separately. If this number is lower than the calculated threshold for node, then the sensor node becomes a cluster head.

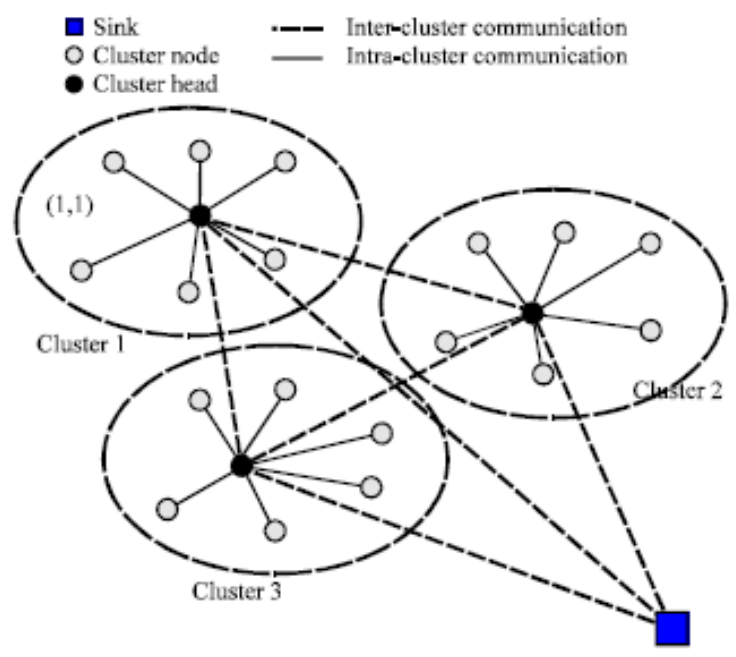

Fig 2: Clustering of sensor nodes

\subsubsection{Cluster formation}

In the cluster formation process each cluster heads broadcast a join message within the sensing field. On reception of a join message each non cluster head sensor node decides to join the cluster head, if it received the join messages more than one then sensor node join the nearest cluster head. After a constant time interval cluster head received join request messages from non-cluster head sensor nodes. It creates a TDMA schedule for data transmission within the cluster and sends to its cluster members.

\subsection{Steady State Phase}

Steady state phase contains a data aggregation and data reporting phase.

\subsubsection{Data aggregation}

In steady state phase, each sensor node senses the physical environment and send sensed data to respective cluster head in the predefined time slot. Each cluster head waits fixed time interval to receive data from its respective cluster member. After this cluster head aggregate sense data.

\subsubsection{Data reporting}

After data aggregation cluster head sends aggregated data to its respective advance $\mathrm{CH}$. If there is any other cluster head node on the way to advance the cluster head, then it send data using multi-hop. Cluster head sends data to nearest cluster head, which send data to advance cluster head. The same procedures apply between advance and super cluster heads. Each super cluster head receives data from its advance cluster heads and aggregated it then sends this data to the base station using multi-hop communication. 


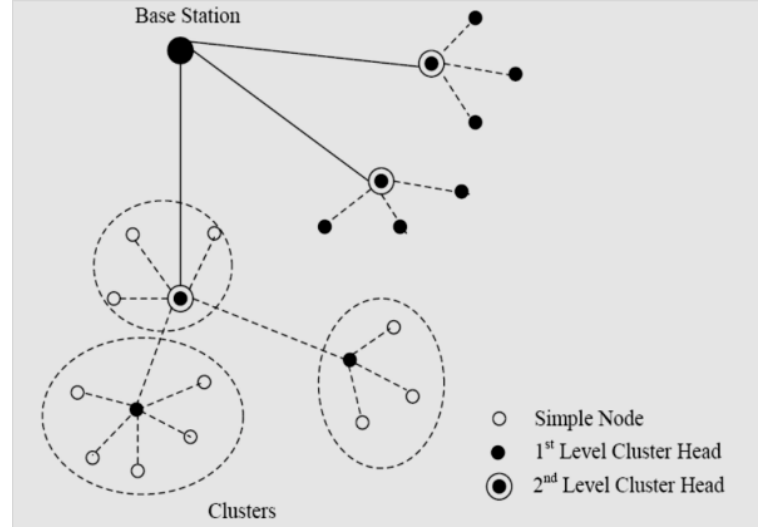

Fig 3: Cluster-based Hierarchical Model

\section{ALGORITHM}

This algorithm includes cluster head selection and cluster formation process. In the cluster head selection process each sensor node chooses a random number between 0 and 1separately.If this number is lower than the calculated thresholdT (i)for node $i$, then the sensor node $i$ become a cluster head.

The threshold T (i) is given by

$\mathrm{T}(\mathrm{i})=\left(\frac{\mathrm{P}}{(1-\mathrm{P} \times \bmod (\mathrm{r}, \operatorname{round}(1 / \mathrm{P})))}\right)(1)$

Where

$\mathrm{P}$ is the optimal probability for intial cluster head

ris the current round

The probability $\mathrm{P}$ for sensor nodes may vary depends on its type;

For heterogeneous sensor node the probability is

$\mathrm{P}_{\mathrm{S}}=\left(\mathrm{P} *(1+\beta) /\left(1+\mathrm{m}_{\mathrm{a}} *\left(\alpha+\beta * \mathrm{~m}_{\mathrm{s}}\right)\right)\right)(2)$

For heterogeneous sensor node the probability is

$\mathrm{P}_{\mathrm{A}}=\left(\mathrm{P} *(1+\alpha) /\left(1+\mathrm{m}_{\mathrm{a}} *\left(\alpha+\beta * \mathrm{~m}_{\mathrm{s}}\right)\right)\right)(3)$

For normal sensor node the probability is

$\mathrm{P}_{\mathrm{N}}=\left(\mathrm{P} /\left(1+\mathrm{m}_{\mathrm{a}} *\left(\alpha+\beta * \mathrm{~m}_{\mathrm{s}}\right)\right)\right)(4)$

Where $\alpha$ is a constant represent the time of energy level of advance node. Bis a constant represent the time of energy level of super node. $\mathrm{m}_{\mathrm{s}}$ is a constant represent the percentage of super node. $\mathrm{m}_{\mathrm{a}}$ is a constant represent the percentage of advance

\section{SIMULATION RESULT}

The algorithm described in Section 4 has been simulated for network size $100 \mathrm{x} 100 \mathrm{~m}$ square units. clustering algorithm is applied on sensor networks with 100 sensors distributed uniformly in a square area of 100sq.Units. Without loss of generality, Transmitter/Receiver electronics $\mathrm{E}_{\text {elec }}$. The value is assigned by the nodes in the normal node $0.5 \mathrm{~J}$ are used super nodes and advance nodes have $\mathrm{m}=0.1, \mathrm{~m} 1=0.1$. The message is transmitted for the 4000 bit of size. Data aggregation is about to $5 \mathrm{nj} / \mathrm{bit} / \mathrm{report}$ and reference distance do $=87$ meter.

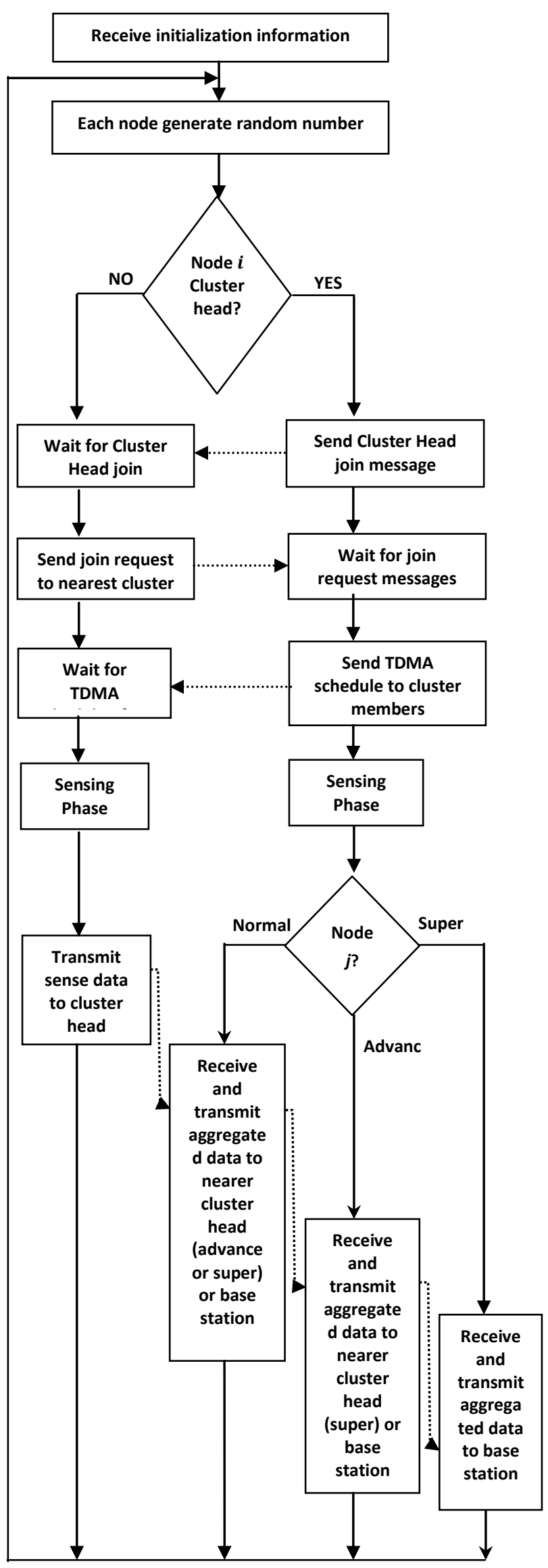

Fig 4: The Flow chart of HACA 
Table 1 Simulation parameters of HACA

\begin{tabular}{|c|c|c|}
\hline \multicolumn{2}{|c|}{ Parameter } & Value \\
\hline \multicolumn{2}{|c|}{ Network size } & $\left(100 \times 100\right.$ meter $\left.^{2}\right)$ \\
\hline \multicolumn{2}{|c|}{ Number of sensor node(n) } & 100 \\
\hline \multicolumn{2}{|c|}{ Base station position } & (100 meter, 100 meter) \\
\hline \multirow{3}{*}{$\begin{array}{l}\text { Initial } \\
\text { energy }\end{array}$} & $\begin{array}{c}\text { Super } \\
\text { heterogeneous } \\
\text { node }\end{array}$ & $1.5 \mathrm{~J}$ \\
\hline & $\begin{array}{c}\text { Advance } \\
\text { heterogeneous } \\
\text { node }\end{array}$ & $1 \mathrm{~J}$ \\
\hline & Normal node & $0.5 \mathrm{~J}$ \\
\hline \multicolumn{2}{|c|}{$\begin{array}{l}\text { Percentage of super } \\
\text { heterogeneous node }\end{array}$} & $\mathrm{m}=0.1$ \\
\hline \multicolumn{2}{|c|}{$\begin{array}{l}\text { Percentage of advance } \\
\text { heterogeneous node }\end{array}$} & $\mathrm{m}_{1}=0.1$ \\
\hline & $\mathrm{P}$ & 0.1 \\
\hline \multicolumn{2}{|c|}{ Transmitter/Receiver } & $50 \mathrm{nj} / \mathrm{bit}$ \\
\hline \multicolumn{2}{|c|}{ Data aggregation $\left(\mathrm{E}_{\mathrm{DA}}\right)$} & $5 \mathrm{nj} / \mathrm{bit} /$ report \\
\hline \multicolumn{2}{|c|}{ Reference distance $\left(\mathrm{d}_{0}\right)$} & 87 meter \\
\hline \multicolumn{2}{|c|}{ Transmit amplifier $\epsilon_{\mathrm{fs}}$} & $10 \mathrm{pJ} / \mathrm{bit} / \mathrm{meter}^{2}$ \\
\hline \multicolumn{2}{|c|}{ Transmit amplifier $\epsilon_{\mathrm{mp}}$} & $0.0013 \mathrm{pJ} / \mathrm{bit} / \mathrm{meter}^{4}$ \\
\hline \multicolumn{2}{|c|}{ Message size (l) } & 4000 bits \\
\hline
\end{tabular}

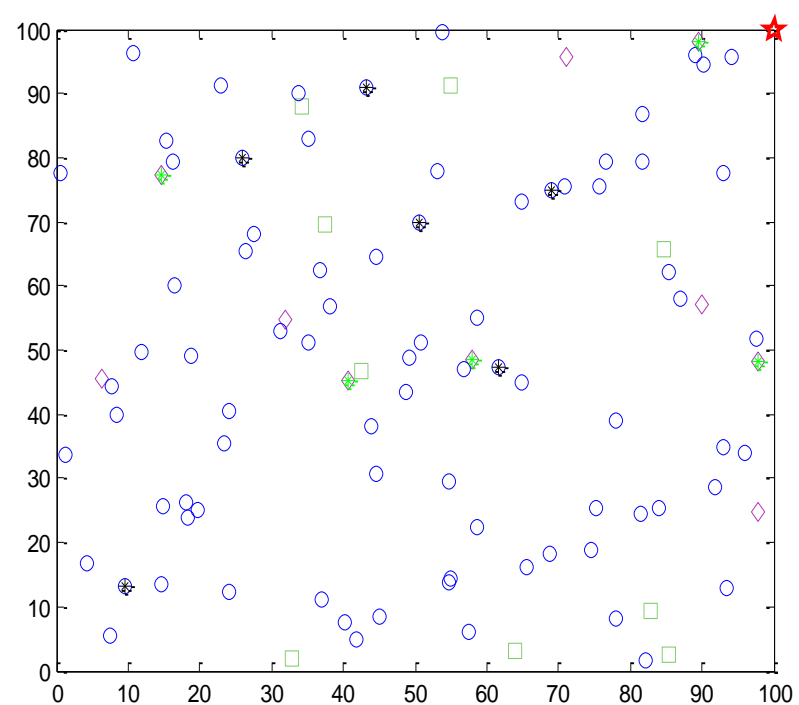

Fig 5: The wireless sensor network deployment

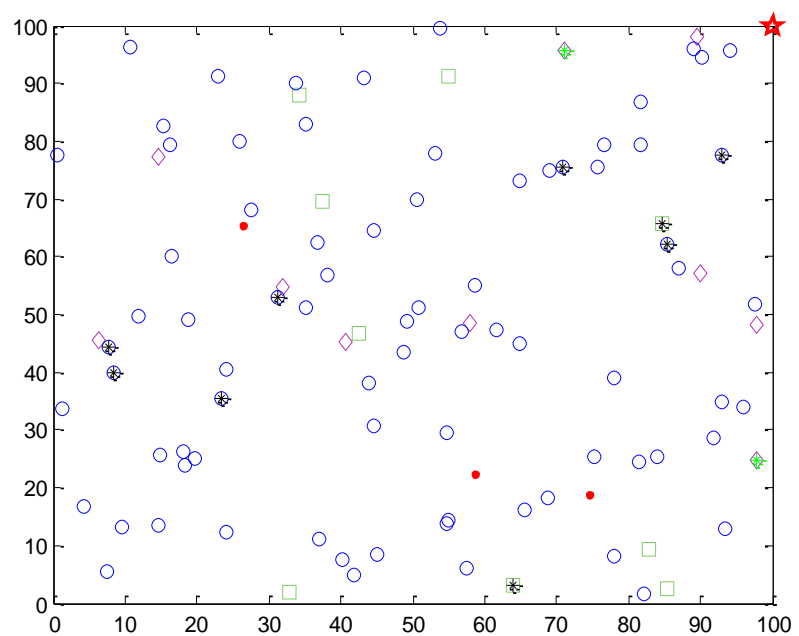

Fig 6:First node dies

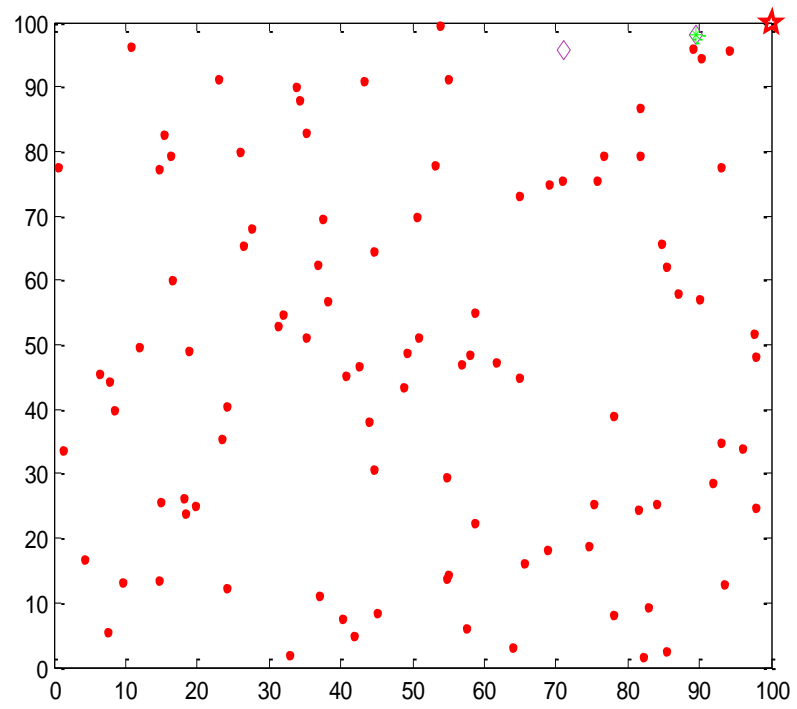

Fig 7: Almost all the node die

Sensor nodes are deployed in $100 \times 100$ geographical area. The blue circleis the normal sensor node, green square is advance sensor nodes and violet diamond is the super sensor node. The base station positioned at $100 \mathrm{X} 100$ and symbolically by a red star as shown in figure 5 . The Figure 6 shows that the first node die but other sensor nodes are still have energy to oprate. At the end super node remains alive when other sensor nodes are exhust there energy as shown in figure 7 .

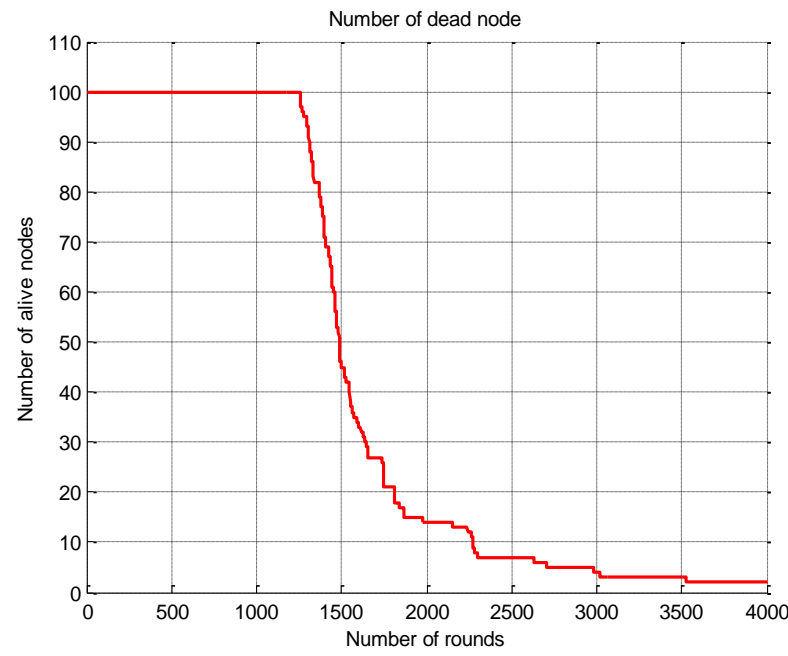

Fig 8: The stability period and network life time of WSN

Figure 8 shows the network stability period and network lifetime of HACA. The HACA is simulated in $100 \times 100 \mathrm{~m}^{2}$ sensing area where 100 senor motes are spreads and base station positioned at $100 \mathrm{~m}, 100 \mathrm{~m}$. The packet length is 4000 bits for this simulation. The stable region of HACA is about 1256 rounds. The network lifespan of HACA is more than 4000 rounds. The observation has been made that the HACA protocol is best in the clustering protocols because it has good stability period as well as higher network lifetime. 


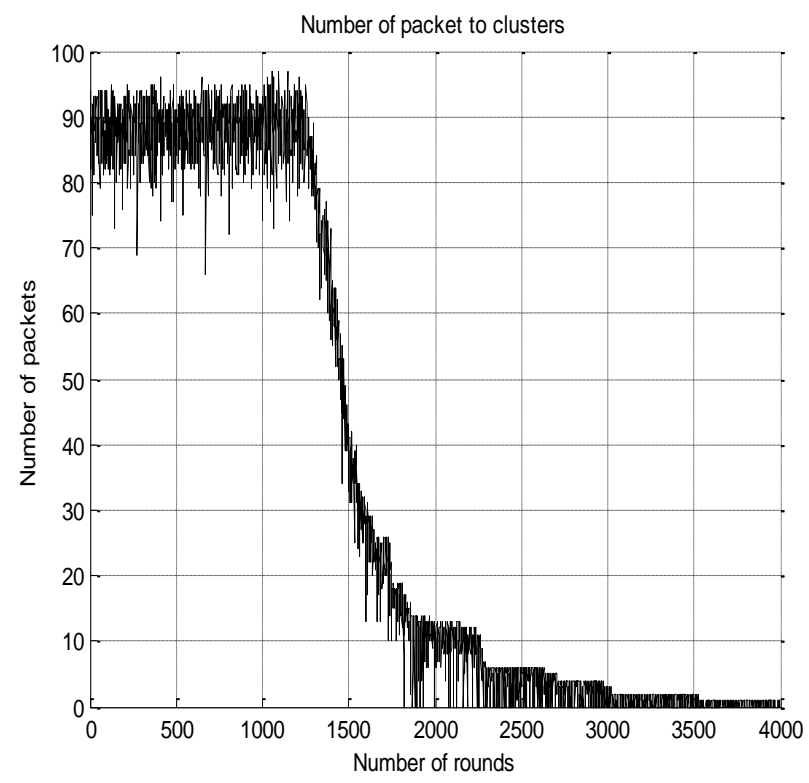

Fig 9: The number of packet transmission within clusters in rounds

The figure shows the number of packets received at the cluster head in HACA protocol.
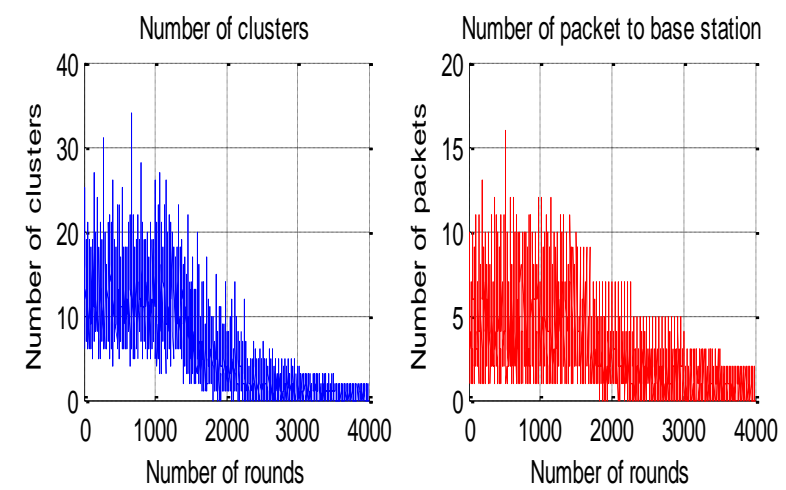

Number of packet to advance cluster heads Number of packet to super cluster heads
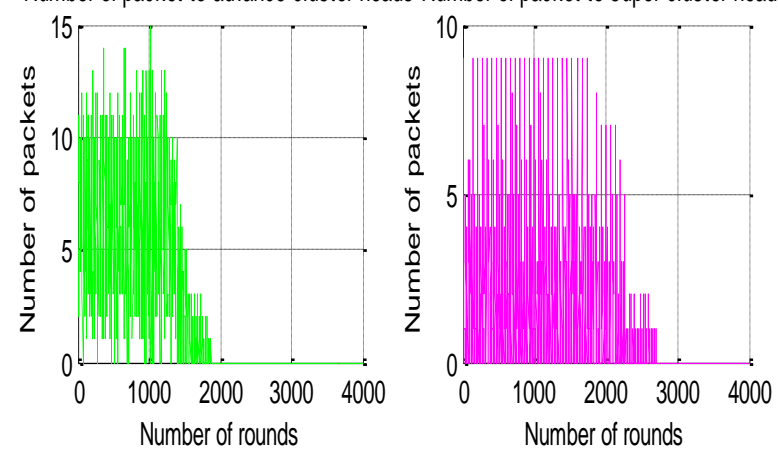

Fig 10: The number of packets sends to base station and cluster head nodes in rounds

The figure 10 shows the number of packets received at the base station in HACA protocol, which is up to 16 packets, but the number of packets transmitted to the base station is lower than the clusters formed in the field (which is up to 33).

\section{CONCLUSION}

This paper presents a distributed algorithm for organizing sensors into a hierarchy of clusters with an objective of minimizing the total energy spent in the system to communicate the information gathered by these sensors to the information-processing center.HACA is very simple to implement in the sensor nodes. This is a dynamic protocol, it will work automatically over no prior distribution of the levels of energy in the nodes.

\section{REFERENCES}

[1] Heinzelman, Wendi Beth. "Application-specific protocol architectures for wireless networks". Diss. Massachusetts Institute of Technology, 2000.

[2] Younis, Ossama, and Sonia Fahmy. "HEED: a hybrid, energy-efficient, distributed clustering approach for ad hoc sensor networks." Mobile Computing, IEEE Transactions on 3.4 (2004): 366-379.

[3] Smaragdakis, Georgios, Ibrahim Matta, and AzerBestavros. SEP: A stable election protocol for clustered heterogeneous wireless sensor networks. Boston University Computer Science Department, 2004.

[4] Amin M Abbosh, David V Thiel, "Energy Efficient Wireless Sensor Networks", IEEE International Conference on Signal Processing and Communications (ICSPC 2007), 24-27 November 2007.

[5] Elshakankiri, Maher N., Mohamed N. Moustafa, and Yasser H. Dakroury. "Energy Efficient Routing Protocol for Wireless Sensor Networks." Intelligent Sensors, Sensor Networks and Information Processing, 2008. ISSNIP 2008. International Conference on. IEEE, 2008.

[6] Wang, Guitang, et al. "The Clustering Algorithm of Wireless Sensor Networks Based on Multi-hop between Clusters." Computer Science and Information Engineering, 2009 WRI World Congress on. Vol. 3. IEEE, 2009.

[7] Gupta, Gaurav, and Mohamed Younis. "Load-balanced clustering of wireless sensor networks." Communications, 2003. ICC'03. IEEE International Conference on. Vol. 3. IEEE, 2003.

[8] Kumar, Dilip, Trilok C. Aseri, and R. B. Patel. "EEHC: Energy efficient heterogeneous clustered scheme for wireless sensor networks." Computer Communications 32.4 (2009): 662-667.

[9] Mahajan, Shilpa, and Jyoteesh Malhotra. "Energy efficient control strategies in heterogeneous wireless sensor networks: a survey." Energy 14.6 (2011).

[10] Aseri, T. C. "Performance Analysis of Cluster based Routing Protocols in Heterogeneous Wireless Sensor Network." International Journal of Computer Applications 83 (2013). 